\title{
Maximum entropy economics: where do we stand?
}

\author{
Ellis Scharfenaker ${ }^{1, a}$ and Jangho Yang $^{2}$ \\ ${ }^{1}$ University of Utah, Salt Lake City, UT, USA \\ ${ }^{2}$ University of Oxford, Oxford, UK
}

Received 24 February 2020

Published online 7 July 2020

Economists have shown an increasing interest in statistical physics and information theory, exploring how entropy concepts can provide useful insights for important economic problems that standard approaches have not been able to satisfactorily deal with. The Maximum Entropy approach (MaxEnt) has been applied to a general equilibrium macro modeling (Rational Inattention), a decision theory with an aggregate feedback (Quantal Response Statistical Equilibrium), econometrics (Info-Metrics), and the analysis of the distribution of micro data such as the returns on capital, the growth rate of firm's size, Tobin's q, asset price, productivity change, and individual income \& wealth distribution. The entropic approach captures the endogenous fluctuations of the economic system along with its central tendency, posing a methodological challenge to the standard economic theory based on the notion of equilibrium as a fixed state such as Walrasian market clearing equilibrium. This methodological advantage over the standard approaches and its successful applications to the real economic data have allowed the information theoretic economic analysis to emerge as a distinct field within economics and have attracted many students and scholars to this filed.

This special issue brings together a diverse group of thinkers under the label Maximum Entropy Economics. While the application of the maximum entropy principle to the study of economic phenomena is without doubt an integral component to econophysics, much work has been done towards moving away from the study and application of stochastic processes originating in statistical physics to economic and financial systems as is often the mainstay of the econophysics literature [1-3]. A significant portion of this work is also being done by economists that reject many of the neoclassical closures. Because the use of Shannon's information theory and the Maximum Entropy Principle of Inference has shed new light on a wide range of economic problems we consider Maximum Entropy Economics to be a more encompassing term that includes the work of economists across the theoretical spectrum as well as those in the physical sciences. We give a brief overview of the contributions within this special issue.

Scharfenaker and Yang [4] detail the history of statistical thinking in economics and argue for the importance of a statistical methodology in economic theory. They argue that the statistical thinking foundational to nineteenth century political economic thought was abandoned by the marginalist approach leading to contemporary problems of the articulation of theory and measurement in economics. While

\footnotetext{
a e-mail: ellis.scharfenaker@economics.utah.edu
} 
econophysics has attempted to address these issues with the use of physical analogy and application of methods from statistical mechanics, they contend that is has fallen short in its scope and interpretive aims. They outline a broader program for the application of the the maximum entropy principle in economics based on information theory.

Duncan Foley [5] lays out the theoretical justification of utilizing elements of information theory in developing economic models. He argues that the assumption of payoff maximizing agents with complete and consistent preferences in the standard economic model is fundamentally inconsistent with widely-observed human behavior that exhibits a substantial degree of indeterminacy and inconsistency. Foley proposes a maximum entropy model of entropy-constrained agents and shows that a wide range of economic phenomena such as endowment effect, loss-aversion, and noise trading, can be understood as a consequence of entropy-constrained behavior.

Kanbur and Venkatasubramanian [6] discuss a micro-foundation of the maximum entropy approach in economics. They argue that many maximum entropy models in econophysics literature do not realize the duality between entropy maximization and payoff/utility maximization. Using occupation/income distribution as an example, they demonstrate such dualism and show that occupational choice equilibrium for individuals with a certain type of preference is equivalent to the maximization of entropy functionals under constraints on the distribution of individuals across occupations.

Paulo dos Santos [7] discusses foundations of maximum entropy approach in economic systems focusing on socio-combinatorial characterizations of statistical equilibrium. He argues that since economic systems are shaped by complex interactions between large numbers of individual members, a combinatorial approach to potential equilibrium distribution rather than detailed descriptions of the microscopic dynamics is most suitable for economic analysis. He compares the maximum entropy approach to two classes of individualist-reductionist characterizations of statistical equilibria: Micro-econometric cross-sectional and drift-diffusion models.

Several contributors discuss the maximum entropy principle in a broader context of complexity economics. Yang and Carro [8] compare the maximum entropy approach to agent-based modeling. They argue that these two methods can complement each other, providing a powerful conceptual/empirical tool for the analysis of complex economic problems. Using two economic models, they show that the maximum entropy approach can guide the development of first-approximation agent-based models, which, in turn, can be used for tracking irreversible and out-of-equilibrium dynamics and thus improving on the original maximum entropy model. Similarly, Özlem Ömar [9] compares and contrasts various complex economic approaches to modeling housing markets: Log Periodic Power Law Models, Heterogenous Agent Models, and Quantal Response Statistical Equilibrium Models (QRSE). She describes QRSE models as the most promising to market fluctuation since it introduces a "general framework" for equilibrium where the conventional market-clearing equilibrium exists just as a special state of the economic systems. Schneider and Scharfenaker [10] give an overview of various finite mixture models used in econophysics. They discuss how the finite mixture model can be understood from the statistical equilibrium perspective and test several mixture models using US income data.

Finally, the special issue includes two important contributions that provide a critical assessment of the maximum entropy approach in economics. Reddy [11] appraises maximum entropy reasoning in terms of scientific explanation by contrasting the ergodic with information theoretic perspective or what he calls the "processual" and "epistemic" views. Favoring "well specified processes" demonstrates how the ergodic view can generate equilibrium outcomes in a stochastic model of arbitrage dynamics that are entropy maximizing. Shaikh [12] similarly argues that economic phenomena 
should be derived as the result of explicit well-defined economic processes rather than as entropy maximizing processes.

Semieniuk and Weber [13] discuss recent work of econophysicists on the maximum entropy approach to the inequality in energy consumption. They argue that choosing an accounting framework is a convention, not information that can be used in the traditional sense in maximum entropy reasoning. The maximum entropy approach has difficulty picking the "correct" accounting convention, as there may be more than one or possibly none. They show that predictions based on information theoretic maximum entropy reasoning are sensitive to the choice of social accounting.

We hope that this volume can contribute to constructive discussions on the maximum entropy economics and stimulate further results in the field.

Publisher's Note The EPJ Publishers remain neutral with regard to jurisdictional claims in published maps and institutional affiliations.

\section{References}

1. R.N. Mantegna, H.E. Stanley, An Introduction to Econophysics: Correlations and Complexity in Finance (Cambridge University Press, Cambridge, UK, 1999)

2. J.L. McCauley, Dynamics of Markets: The New Financial Economics (Cambridge University Press, 2009)

3. A. Chatterjee, S. Yarlagadda, B.K. Chakrabarti, eds., Econophysics of Wealth Distributions: Econophys-Kolkata I, New Economic Windows (Springer-Verlag Mainland, 2005)

4. E. Scharfernaker, J. Yang, Eur. Phys. J. Special Topics 229, 1577 (2020)

5. D.K. Foley, Eur. Phys. J. Special Topics 229, 1591 (2020)

6. R. Kanbur, V. Venkatasubramanian, Eur. Phys. J. Special Topics 229, 1661 (2020)

7. P.L. dos Santos, Eur. Phys. J. Special Topics 229, 1603 (2020)

8. J. Yang, A. Carro, Eur. Phys. J. Special Topics 229, 1623 (2020)

9. Ö. Ömer, Eur. Phys. J. Special Topics 229, 1715 (2020)

10. M.P.A. Schneider, E. Scharfenaker, Eur. Phys. J. Special Topics 229, 1685 (2020)

11. S. Reddy, Eur. Phys. J. Special Topics 229, 1645 (2020)

12. A. Shaikh, Eur. Phys. J. Special Topics 229, 1675 (2020)

13. G. Semieniuk, I.M. Weber, Eur. Phys. J. Special Topics 229, 1705 (2020) 